\title{
Synthetic TLR4 agonist as a potential immunotherapy for melioidosis
}

\author{
Zi Yan Tan, Alvin K. L. Khah, Siew Hoon Sim, Vidhya Novem, Yichun Liu, \\ Gek-Yen Gladys Tan *
}

Host-Pathogen Interaction Laboratory, Defence Medical \& Environmental Research Institute, DSO National Laboratories, Singapore City, Singapore; ${ }^{*}$ Corresponding Author: tgekyen@dso.org.sg

Received 29 October 2012; revised 30 November 2012; accepted 10 December 2012

\begin{abstract}
Melioidosis, an infection caused by Gram-negative Burkholderia pseudomallei (Bp), has high clinical recurrence and mortality rates associated with pneumonia and sepsis. With the limitations in current therapeutic options and the lack of available human vaccines, development of novel countermeasures against Bp infection is vital. In this study, we evaluated the efficacy of an aminoalkyl glucosaminide 4-phosphate (AGP), a synthetic toll like receptor 4 agonist (CRX-527), in conferring protection against melioidosis in a murine model. Survival data showed $66 \%$ of mice treated with AGP prior to lethal intranasal Bp challenge survived and presented no signs of illness over a 3 months period. In contrast, all control mice succumbed to infection within 4 days. Kinetic study on organ bacterial burden demonstrated mice treated with AGP had dramatically reduced bacterial loads in both the lungs and spleens as compared to control mice. Notably, all but one AGP-treated mouse had no Bp growth in the blood as compared to overwhelming bacteraemia found in all control mice. The protective effect of CRX-527 was associated with a transient increase in pulmonary cytokinel chemokine levels, which boosted the host's innate immunity. This enabled rapid clearance of the pulmonary and systemic bacterial burden and prevented the development of sepsis. This study demonstrated the potential use of TLR4 agonist as a prophylactic immunotherapy in preventing melioidosis.
\end{abstract}

Keywords: Melioidosis; AGP; Pneumonia; Sepsis; TLR4 Agonist; Bukholderia pseudomallei

\section{INTRODUCTION}

Burkholderia pseudomallei (Bp), the causative agent of Melioidosis, is a Gram-negative environmental saprophyte endemic in South-East Asia and northern Australia [1]. However, Bp has gained significant attention in recent years due to its increasing global distribution $[2,3]$ as well as its classification as a potential biothreat agent [4].

Inhalation has been recognized as an important route of infection in melioidosis although Bp can be transmitted through other routes such as percutaneous inoculation and ingestion [5]. Therefore, melioidosis can virtually affect any organ and is potentially fatal, with a mortality rate of up to $50 \%$ in endemic regions such as Thailand [6, 7]. It can also present with a wide range of clinical manifestation, which is thought to be determined by the host's immune response [4,8]. Currently, there is no available vaccine for melioidosis and present antibiotic treatment is not fully effective, with frequent relapses occurring $[8,9]$. Although manipulating the host's immune system pre/post-infection with the use of granulocyte colony stimulating factor (G-CSF) or interferongamma (IFN- $\gamma$ ) has been proposed [10-12] as treatments for melioidosis, clinical benefits of such therapy are limited and need to be re-evaluated. Therefore, efforts have recently been directed at developing novel immunotherapeutic strategies to prevent or treat melioidosis.

Toll-like receptors (TLRs) are important arms of the innate immune system. These receptors recognize pathogen-associated molecular patterns (PAMPs) and provide a first line of defence against infectious organisms. Lipopolysaccharide (LPS), present in the outer membrane of Gram-negative bacteria, is a natural agonist for TLR4 signalling [13]. However, Bp is thought to evade the host immune system through its poorly recognized lipid A component within its LPS [14]. In addition, the release of secondary 2-hydroxymyristoyl residue in its lipid A can potentially interfere with MyD88-independent (TRIF) signalling in macrophages as it gets converted into 2-hydroxymyristoyl CoA, a potent inhibitor against N-myristoylation of TRIF-related adaptor molecule (TRAM) $[15,16]$.

Although administration of LPS or lipid A as prophy- 
lactic treatment has been suggested, its use has been precluded by its high toxicity and pyrogenicity $[17,18]$. The use of lipid A mimetics such as aminoalkyl glucosaminide 4-phosphates (AGPs) has been proposed as these molecules are less toxic than LPS but equally immunostimulatory [19]. Recent animal studies have yielded promising results, demonstrating prophylactic protection against pneumonic plague and tularaemia with AGPs [18, 20]. Hence, it can be hypothesized that pre-exposure administration of an AGP, particularly CRX-527, a potent inducer of MyD88-dependent TLR4 signalling [21], could protect the host against Bp infection. Therefore, this study evaluated the induction of protective immunity, bacterial clearance and host survivability with CRX-527 pre-exposure treatment in a murine model of pulmonary melioidosis.

\section{MATERIALS AND METHODS}

\subsection{Animals}

Specific-pathogen-free male C57BL/6 mice, 6-8weeks, were obtained from Biological Resource Centre (A*Star, Singapore). All animals were housed in individuallyventilated cages (Techniplast, Italy) and were permitted ad libitum access to sterile food and water in a Biosafety Level Three Animal Facility. Anaesthesia, Ketamine (50 $\mathrm{mg} / \mathrm{kg}$ ) and Xylazine $(5 \mathrm{mg} / \mathrm{kg})$, was administered intraperitoneally prior to treatment, infection and blood collection. All experimental procedures were performed in accordance to the guidelines approved by the Institutional Animal Care and Use Committee, DSO National Laboratories (DSO/IACUC/10/92).

\subsection{Optimal Dose for CRX-527 Treatment}

CRX-527 was provided by GlaxoSmithKline Vaccines (Hamilton, Montana, USA) as a $0.934 \mathrm{mg} / \mathrm{mL}$ solution in $2 \%$ aqueous glycerol and stored at $4{ }^{\circ} \mathrm{C}$. To determine the optimum dose, CRX-527 was diluted in $2 \%$ glycerol at varying doses $(20 \mu \mathrm{g}, 10 \mu \mathrm{g}, 5 \mu \mathrm{g}, 2.5 \mu \mathrm{g}$ and $1.25 \mu \mathrm{g})$. Mice were anaesthetised and $40 \mu \mathrm{l}$ of CRX-527 was administered intranasally/intrapulmonary $(20 \mu \mathrm{l} /$ nare $)$. Controls were given either sterile PBS or $2 \%$ glycerol.

\subsection{Bp Strain and Growth Conditions}

B. pseudomallei clinical strain K96243 from glycerol stock was subcultured onto Trypticase Soy Agar (TSA) (Becton Dickinson, USA) and allowed to grow for 48 hours at $37^{\circ} \mathrm{C}$. A single colony was then cultured onto another TSA plate for 24 hours prior to infection experiments.

\subsection{Bp Infection}

For infection experiments, colonies from TSA agar were suspended in sterile PBS to an $\mathrm{OD}_{600}$ of 1.02 (equivalent to $\sim 6 \times 10^{8} \mathrm{CFU} / \mathrm{ml}$ ) and diluted to the desired concentration. Mice were anaesthetised and the Bp suspension was then given intranasally $(50 \mu \mathrm{l})$ into the mice. Controls were given $50 \mu 1$ sterile PBS. Mice were monitored for their survival. Aliquots of the suspension were plated out on TSA plates in duplicates to determine the number of viable bacteria inoculated into the mice.

\subsection{Organ Bacterial Load}

Spleens, lungs and livers were excised and placed in 2 $\mathrm{ml}$ sterile PBS. Organs were homogenized in a $70 \mu \mathrm{m}$ nylon mesh sieve, after which they were serially diluted, plated out on Ashdown agar and incubated at $37^{\circ} \mathrm{C}$ for at least 48 hours to determine organ bacterial load. Colonies were counted from plates and the mean colony counts were derived to give bacterialload in the different organs.

\subsection{Quantification of Cytokines and Chemokines}

The plasma and organ homogenates were assayed for interleukin-6 (IL-6), interferon- $\gamma$ (IFN- $\gamma$ ), tumour necrosis factor- $\alpha$ (TNF- $\alpha$ ) and granulocyte-monocyte colony stimulating factor (GM-CSF), monocyte chemotactic protein-1 (MCP-1), RANTES, macrophage inflammatory protein-1 $\beta$ (MIP-1 $\beta$ ) and monokine induced by gamma interferon (MIG) using FACS Array Bioanalyser (Becton Dickinson, USA) and CBA flex sets (Becton Dickinson, USA), as per manufacturer's instructions. Samples of lungs, spleen and plasma of uninfected mice were used as negative control to obtain basal levels.

\subsection{Statistical Analysis}

All graphs and statistical analysis were generated with Graphpad PRISM. $\mathrm{p}$ value of $<0.05$ was considered statistically significant.

\section{RESULTS}

\subsection{Intranasal Administration of CRX-527 Enhanced Survival against Melioidosis}

The prophylactic protection of CRX-527 has been demonstrated in various mice models against other Gram-negative bacterial infection when delivered 48 hours prior to bacterial challenge $[18,20]$. Our initial experiment was aimed at determining the optimum dose of CRX-527 administered via intranasal/intrapulmonary route 48 hours prior to lethal $\mathrm{Bp}$ intranasal challenge (28x 10-day $\mathrm{LD}_{50}$ ). Groups of mice were pre-treated with five individual doses of CRX-527 $(20 \mu \mathrm{g}, 10 \mu \mathrm{g}, 5 \mu \mathrm{g}$, $2.5 \mu \mathrm{g}$ and $1.25 \mu \mathrm{g}$ ) and monitored daily for signs of 
sickness before and after Bp challenge. Mice pre-treated with $20 \mu \mathrm{g}$, presented with drug related side-effects such as mild lethargy and piloerection before Bp challenge. In comparison, mice pre-treated with lower doses of CRX527 appeared healthy.

Mice pre-treated with either 5 or $10 \mu \mathrm{g}$ of CRX-527 did not develop obvious signs of sickness in comparison to other treated counterparts post Bp challenge. The survival rate was determined at day 90 post challenge (Figure 1) to further confirm the optimum protection dosage of CRX-527. Untreated mice demonstrated 100\% mortality by day 4 post infection. Pre-treatment with $1.25 \mu \mathrm{g}$, $2.5 \mu \mathrm{g}$ or $20 \mu \mathrm{g}$ CRX-527 presented with a survival rate of $16 \%, 50 \%$ and $33 \%$ respectively at day 90 post Bp challenge. In contrast, mice pre-treated with either $5 \mu \mathrm{g}$ $(\mathrm{p}<0.05)$ or $10 \mu \mathrm{g}(\mathrm{p}<0.001) \mathrm{CRX}-52748 \mathrm{~h}$ prior to the high lethal dose of Bp challenge prolonged time to death and demonstrated significant increase in survivability (66\%) at day 90 (Figure 1).

\subsection{Pre-Exposure Treatment with CRX-527 Primed the Host's Innate Immune System}

CRX-527 has been previously reported as a potent. synthetic TLR-4 agonist and is able to induce protective innate immune response contributed by its unique chemical structure [22,23] In this study, we measured the pulmonary production of pro-inflammatory cytokines and chemokines in naïve C57BL/6 mice induced with 5 ug CRX-527 against Bp intranasal challenge over a period of 5 days (Figure 2). The results showed a transient increase, with a peak at day 2 , in levels of IL-6, IFN- $\gamma$ and MCP-1 in the lungs before returning to basal level after day 4 post treatment. Additionally, levels of TNF- $\alpha$ increased at 4 hours post treatment and remained elevated until Day 3 (Figure 2). GM-CSF and chemokines such as RANTES, MIG, MIP- $1 \beta$ also presented similar trend (data not shown).

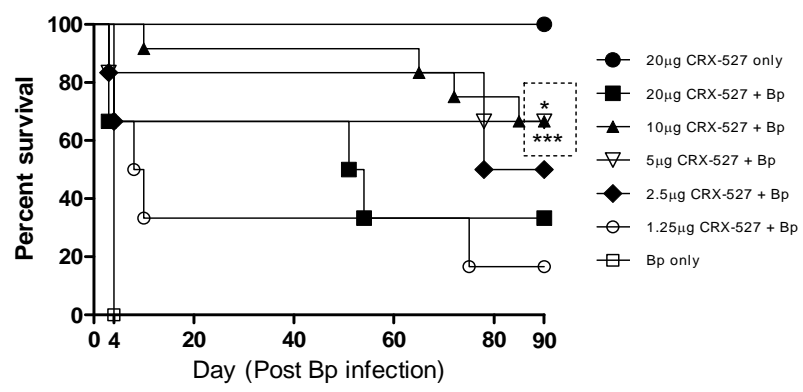

Figure 1. Percentage survival of CRX-527 pre-treated mice at varying doses before intranasal $\mathrm{Bp}$ infection. Mice were treated at 48 hours (h) with various doses of CRX-527 followed by a lethal dose of Bp challenge $\left(1.4 \times 10^{4} \mathrm{CFU}\right.$; $28 \mathrm{x}$ 10-day LD50). Mice were monitored over 90 days to determine survival rate. ${ }^{*} \mathrm{p}<0.05$ and ${ }^{* * *} \mathrm{p}<0.001(\mathrm{n}=6-12)$.
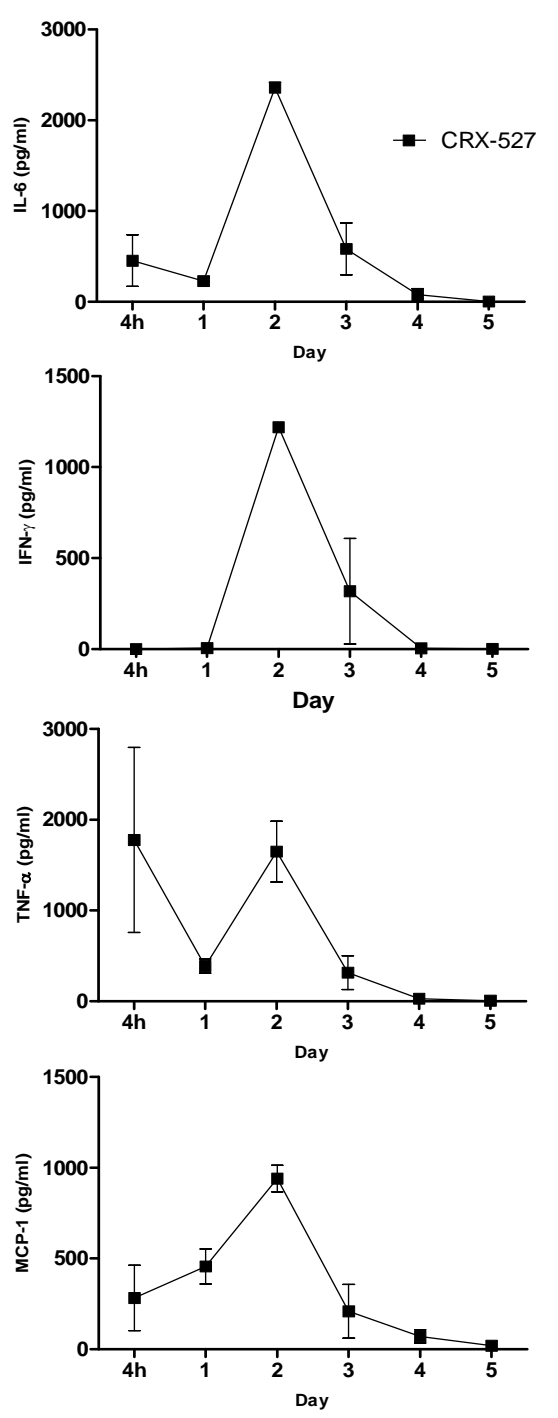

Figure 2. Levels of IL-6, TNF- $\alpha$, IFN- $\gamma$ and MCP-1 in the lungs post CRX-527 administration. Mice were administered with $5 \mu \mathrm{g}$ CRX-527 intranasally and the lungs were excised at different timepoints to determine local levels of various cytokines and chemokines. Data is presented as mean $\pm \operatorname{SEM}(n=4)$.

To further assess the association between the prophylactic effects of CRX-527 and the kinetic changes in levels of cytokines and chemokines, groups of mice were then treated with 5 ug CRX-527 either before ( $-72 \mathrm{~h}$, $-48 \mathrm{~h}$ and $-24 \mathrm{~h})$ or $\operatorname{after}(0 \mathrm{~h}$ and $+24 \mathrm{~h})$ a lethal dose (17x 10-day $\mathrm{LD}_{50}$ ) of Bp challenge (Figure 3 ). Hundred percent survival rate was observed in mice pre-treated at 48 hours with $5 \mu \mathrm{g}$ CRX-527 at day 19 post Bp challenge (versus $0 \%$ in untreated mice). In comparison, mice pre-treatedwith CRX-527 at either -72 or -24 hours presenteda lower survival rate $(83 \%)$ while all mice with post-exposure treatment ( 0 and +24 hours) died at day 3 
(Figure 3). Collectively, data from these studies indicated that $5 \mu \mathrm{g}$ CRX-527 administered intranasally/ intrapulmonary 48 hours prior to Bp intranasal infection was the most effective treatment regime.

\subsection{CRX-527 Decreased Bacterial Colonization and Enhanced Clearance of Bps}

To further elucidate the efficacy of CRX-527, the kinetics of bacterial clearance/colonization in the lungs and spleen post-Bp infection were determined (Table 1). Bp colonization increased significantly $\left(>10^{6}\right.$ CFU/organ) day 2, which resulted in the mice succumbing to infection by day 4 . In contrast, mice pre-treated with CRX527 presented with a significant reduction in Bp hours post infection and were able to clear the bacteria in their lungs by day 13 (Table 1). Furthermore, spleens of

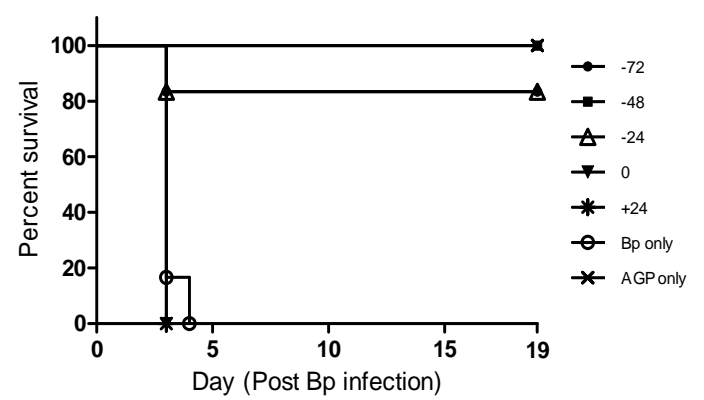

Figure 3. Percentage survival of CRX-527 (5 $\mu \mathrm{g})$ pre-treated mice at varying time points prior/after intranasal Bp infection. Mice were treated either pre- or post Bp challenge $\left(8.5 \times 10^{3} \mathrm{CFU}\right.$; $17 \mathrm{x}$ 10-day LD50) at various time-points. Mice were monitored over 19 days to determine survival rate $(n=6)$.
CRX-527 pre-treated mice harboured lesser Bp as compared to their counterparts from day 1 post-Bp infection, with statistically significant reduction evident by day 2 $(\mathrm{p}<0.001)$ (Table 1).

\subsection{Pre-exposure Treatment with CRX-527 Prevented Cytokine and Chemokine Storm during Bp Infection}

The cytokines and chemokines produced during Bp infection \pm treatment were evaluated. An early induction of IFN- $\gamma$, TNF- $\alpha$ and IL- 6 was observed in lungs of treated mice 4 hours post Bp challenge while the levels. of MCP-1 and GM-CSF in lungs maintained at a basal level throughout the observation period (13 days) (Figure 4).

On the other hand, there were no significantincrease in levels of these cytokines and chemokines in the spleens of treated mice at all time points post $\mathrm{Bp}$ infection.

In contrast, remarkably high levels of IFN- $\gamma$, TNF- $\alpha$, IL-6, GM-CSF and MCP-1were observed in both lungs and spleens of untreated mice (Figure 4) indicating an overwhelming cytokine and chemokine storm in untreated mice in response to pulmonary Bp challenge.

\subsection{CRX-527 Prophylactic Treatment Prevented Onset of Sepsis}

Sepsis arising from pneumonia is an important clinical manifestation associated with high level of mortality in human melioidosis. Therefore, the ability of CRX-527 to prevent sepsis was evaluated in this pneumonic model. Untreated mice were bacteraemic within 4 hours postinfection, reaching a mean blood bacterial load of $10^{4}$ by day $2(\mathrm{p}<0.001)$ (Table 2). Plasma cytokine/chemokine

Table 1. Mean bacterial load in the lungs and spleens of mice post Bp infection (16x 10-day LD50) $(n=4-5)$.

\begin{tabular}{cccccccc}
\hline & \multirow{2}{*}{ Organs } & \multicolumn{5}{c}{ CFU/organ (mean \pm SEM) at different time points } \\
\cline { 3 - 7 } & & $4 \mathrm{~h}$ & $\mathrm{D} 1$ & $\mathrm{D} 2$ & $\mathrm{D} 3$ & D6 & D13 \\
\hline Bp only & Lungs & $>10^{4}$ & $>10^{5}$ & $>10^{6}$ & $>10^{6}$ & - & - \\
CRX-527+Bp & $1210 \pm 387^{\mathrm{a}}$ & $>10^{4}$ & $3964 \pm 2306^{\mathrm{a}}$ & $7203 \pm 4291^{\mathrm{a}}$ & $90 \pm 68$ & 0 \\
Bp only & & 0 & $1011 \pm 989$ & $>10^{6}$ & $>10^{6}$ & - & - \\
CRX-527 + Bp & Spleen & 0 & $336 \pm 336$ & $4 \pm 2^{\mathrm{a}}$ & $237 \pm 119^{\mathrm{a}}$ & $97 \pm 53$ & $2 \pm 2$ \\
\hline $\mathrm{a} p<0.001$. & & & & & &
\end{tabular}

Table 2. Mean bacterial load in the blood of mice post Bp infection (16x 10-day LD50) (n= 4 - 5).

\begin{tabular}{cccccccc}
\hline & & \multicolumn{5}{c}{ CFU/organ (mean \pm SEM) at different time points } \\
\cline { 3 - 7 } & Organs & $4 \mathrm{~h}$ & $\mathrm{D} 1$ & $\mathrm{D} 2$ & $\mathrm{D} 3$ & $\mathrm{D} 6$ & $\mathrm{D} 13$ \\
\hline Bp only & Blood & $38 \pm 38$ & $1264 \pm 833$ & $>10^{4}$ & $>10^{4}$ & - & - \\
CRX-527+ Bp & & 0 & 0 & $0^{\mathrm{a}}$ & $4 \pm 4^{\mathrm{a}}$ & 0 & 0 \\
\hline
\end{tabular}

${ }^{\mathrm{a}} \mathrm{p}<0.001$. 
Lungs
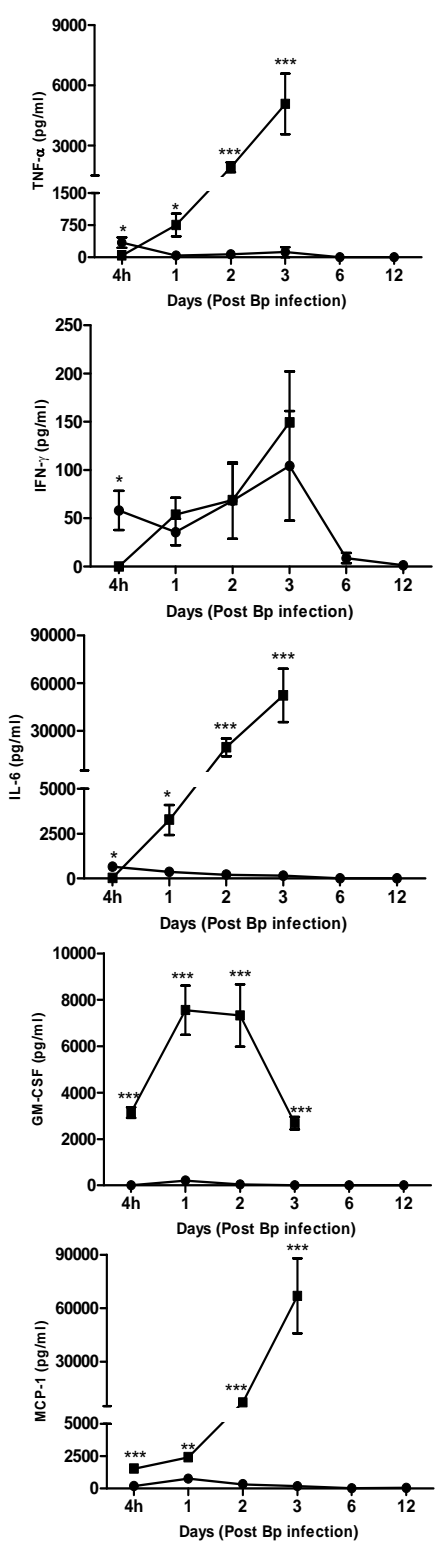

Spleen
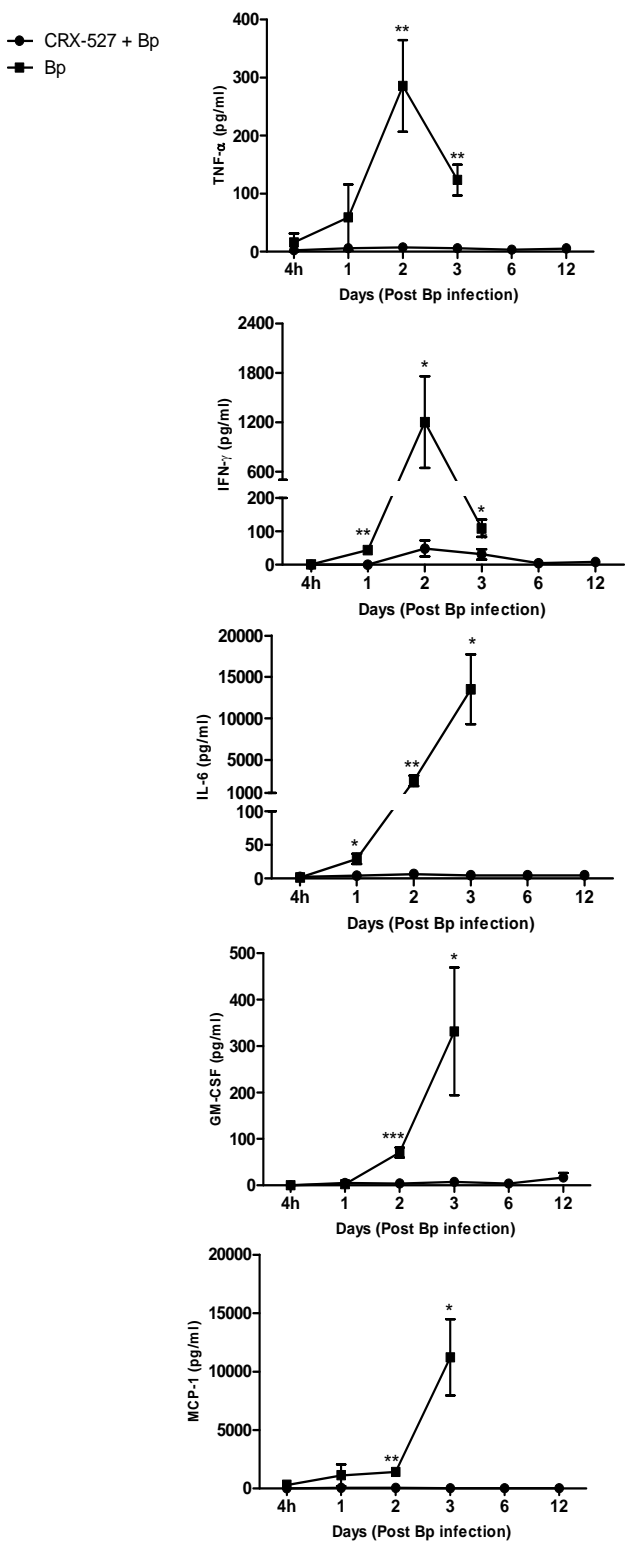

Figure 4. Cytokine/chemokine levels in the lungs and spleens of mice post Bp infection \pm CRX-527 pre-treatment. Mice were either treated with $5 \mu \mathrm{g}$ CRX527 or PBS48 hours prior to Bp infection $(8 \times 103 \mathrm{CFU}$; 16x 10-day LD50). The level of IL- 6 , TNF- $\alpha$, IFN- $\gamma$ GM-CSF and MCP-1 post infection was determined at different time-points. Data is presented as mean $\pm \operatorname{SEM}(n=4-5) .{ }^{*} \mathrm{p}<0.05$, ${ }^{* *} \mathrm{p}<0.01$ and ${ }^{* * * *} \mathrm{P}<0.001$.

levels correlated with the bacterial load in the blood, with significantly elevated levels of IL- 6 andIFN- $\gamma$ beginning day 1 post-infection. TNF- $\alpha$, GM-CSF and MCP-1 levels were also significantly elevated beginning day 2 post-infection (Figure 5).

In comparison, pre-treatment with CRX-527 prevented sepsis. Bp was undetectable in the blood of treated mice at 4 hours, day 1, 2, 6 and 13 post-infection, although 1 out of 4 mice harboured $4 \mathrm{CFU} / \mathrm{ml}$ at day 3 (Table 2).
Plasma levels of IL- 6 , TNF- $\alpha$, GM-CSF, IFN- $\gamma$ and MCP-1 maintained at basal levels throughout the observation period (13 days) (Figure 5).

\section{DISCUSSION}

Relapses and mortality rates from Melioidosis remain high despite appropriate antibiotic therapy. Furthermore, the recent emergence of Bp stains resistant to ceftazidime 

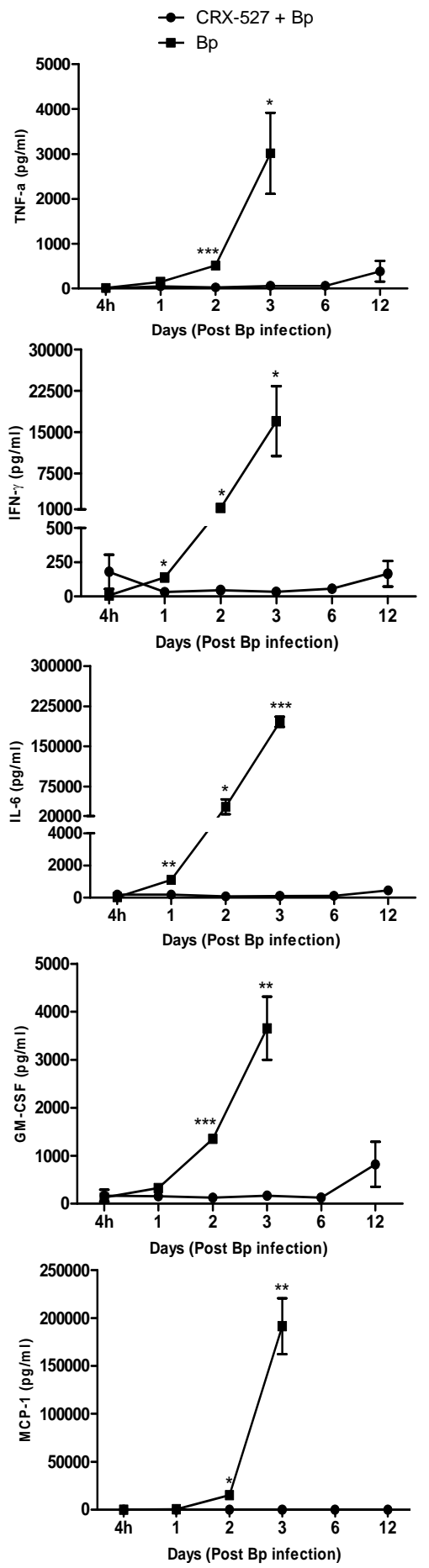

Figure 5. Cytokine/chemokine levels in the plasma of mice post Bp infection \pm CRX-527 pre-treatment. Mice were either pre-treated with $5 \mu \mathrm{g}$ CRX527 or PBS (untreated) 48 hours prior to Bp infection $\left(8 \times 10^{3} \mathrm{CFU}\right.$; $16 \times 10$ day LD50). The level of cytokines post infection was determined at different time-points. Data is presented as mean \pm SEM $(\mathrm{n}=4-5)$. ${ }^{*} \mathrm{p}<0.05,{ }^{* *} \mathrm{p}<$ 0.01 and ${ }^{* * *} \mathrm{P}<0.001$. the first choice of antibiotics for treatment of severe melioidosis, and the lack of available human vaccines strongly necessitate the search for alternative treatment strategies. Recently, immunomodulators such as TLR agonists have gained increasing interest due to their broad spectrum efficacy against a wide range of infectious diseases e.g. tularemia and pneumonic plague $[18,20]$. Results from this study demonstrated significant prophylactic efficacy of CRX-527, a member of the AGP class of synthetic lipid Amimetics, against melioidosis.

A high percentage $(\sim 50 \%)$ of melioidosis patients develop pneumonia either through direct inhalation of Bp or dissemination from extrapulmonary sites $[5,8]$, which can consequently progress to sepsis, septic shock and eventually death $[9,24]$. Hence, intranasal challenge with Bp was chosen as the model to mimic pneumonicmelioidosis in this study. The prophylactic protection of CRX527 presented a dose-dependent fashion, however, administration with $20 \mu \mathrm{g}$ of CRX-527 unexpectedly resulted in 66\% lethality (Figure 1). This could indicate some pyrogenic side effects associated with high dose CRX-527. As a result, the combinatory effects with Bp infection could have perturbed and over stimulated the immune system, resulting in increased tissue damage and death. In contrast, pre-treatment with either 5 or $10 \mu \mathrm{g}$ CRX-527 prolonged time to death, provided.

substantialprotection and survived mice appeared healthy over 90 days post infection (Figure 1). Although $100 \%$ protection was not achieved, it is suggestive that the delay in time to death may provide a window during the acute stage of disease for other medical interventions to be administered against such high dose infection. Repeated experiments showed similar prophylactic protection between 5 and $10 \mu \mathrm{g}$, hence the former was chosen as the optimal dosage for this model to eliminate potential drug associated pyrogenic side effects as described previously.

Optimal protection with CRX-527 was also time-dependent. Pre-treatment with 5ug CRX-527 48 hours prior to Bp (17x 10-day LD50) challenge further demonstrated its prophylactic efficacy, presenting $100 \%$ survival rate 19 days post infection (Figure 3). Notably, significant protection could also be observed with administration 72 or 24 hours prior to Bp challenge, as compared to the untreated group $(p<0.001)$ (Figure 3). However, post exposure treatment did not reduce the lethality of $\mathrm{Bp}$ as all mice died by day 3 . It could be postulated that the synergistic effect of CRX-527 with high dose Bp challenge might exacerbate the infection, resulting in increased inflammation, endotoxic shock and eventually death. Therefore, as a post exposure therapy, a lower dose of CRX-527 or combination with sub-therapeutic antibiotics might improve survivability of the host as seen in Airhart et al., 2008. 
A unique feature of $\mathrm{Bp}$ is the induction of low-level immunological activation associated with the lipid A component within its LPS [14]. This characteristic allows $\mathrm{Bp}$ to remain undetected and facilitates intracellular survival and latency. Therefore, priming of the innate immune system with a strong TLR4 ligand such as CRX527 can aid in the elimination of Bp before allowing it to escape from the host's immune system. Intranasal/ intrapulmonary administration of CRX-527 induced a transient increase, with a peak at day 2 , in intrapulmonary levels of pro-inflammatory cytokines (Figure 2). In addition, levels of TNF- $\alpha$ peaked rapidly at 4 hours post treatment before returning to basal level after 72 hours (Figure 2). This finding is in line with observations reported by Airhart et al. (2008), where CRX-527 treatment led to induction of IFN- $\gamma$ and TNF- $\alpha$, which were shown to reach peak levels by 18 hours and $4-6$ hours respectively. This might suggest that CRX-527 improved bacterial clearance, in part, by promoting the activation and migration of immune cells through binding to TLR $4^{+}$ cells (e.g. epithelial cells, dendritic cells and macrophages) and activating MyD88-dependent and -independent signalling pathways $[22,25,26]$. Another mechanism of protection could be attributed to the priming of neutrophils, which are important for bacterial clearance during melioidosis. This promotes mobilization of secretory vesicles and cytokine release without degranulation and reactive oxygen release [27-29]. Consequently, these processes promoted production of multiple inflammatory cytokines such as TNF- $\alpha$, IL- 6 and IFN- $\gamma$, which induces an early non-specific innate immune response prior to infection. As a result, this provided prophylactic protection against $\mathrm{Bp}$ and contributed to the increased survival rate (Figures 1-3).

Sepsis is a clinical syndrome defined as an overwhelmed systemic inflammatory response to infection [30]. High levels of cytokines such as TNF- $\alpha$, IFN- $\gamma$, IL-6 and MCP-1 are often associated with the severity of sepsis [31-33]. Studies have shown direct correlations between hypercytokinaemia in melioidosis with disease severity and fatal clinical outcome [33-36]. In this study, an early bacterial colonization in the lungs with bacteraemia was observed in untreated mice from 4 hours post infection, suggesting the failure of the innate immune system in preventing the onset of infection. This correlated with increase in levels of proinflammatory cytokines in the spleen, lungs and plasma, with statistical significance by day 2 (Figures 4 and 5, Tables 1 and 2).

In addition, levels of chemokines (MIG and MIP-1 $\beta$ ), which are important for the recruitment of lymphocytes and granulocytes during melioidosis, were also shown to be considerably increased (data not shown). It has been well established that C57BL/6 mice develop a macro- phage dominant immune response during acute melioidosis, which helps control the infection before an adequate adaptive immune response ensue [37,38]. This might explain for the substantial increase in levels of MCP-1 and GM-CSF (Figure 4) observed at 4 hours post-infection in the untreated group, as these cytokines are essential for the recruitment and proliferation of macrophages. Thus, in line with previous studies, untreated mice succumbed to infection as a consequence of overwhelming bacterial colonization/dissemination and the hyperproduction of pro-inflammatory cytokines/chemokines [33,38,39]. Hence, the exacerbated local and systemic inflammatory responses might lead to increased tissue damage and septic shock, which contributed to the death of the host.

In contrast, mice pre-treated with CRX-527 prior to Bp challenge showed significant reduction in organ bacterial load (lung, spleen and blood) as early as 4 hours post infection, with complete bacterial clearance by day 13 (Tables 1 and 2). A combination of three mechanisms could be proposed. Firstly, thesignificantly higher levels of pulmonary IFN- $\gamma$, IL- 6 andTNF- $\alpha$ present at 4 hours post infection (Figure 4) in CRX-527 pre-treated mice provided the immune capability for the host to contain the bacteria upon infection, consequently potentiating rapid clearance of the bacteria before spilling over into the systemic compartment. It has been previously demonstrated that IFN- $\gamma$ plays an obligatory protective role in the resistance against Bp, particularly within the first 24 hours post infection [40,41]. High levels of pulmonary IFN- $\gamma$ at 4 hours post infection could also be vital in controlling the colonization and spread of Bp during the initial phase of infection, hence modulating the inflammatory response and preventing the onset of sepsis.

Secondly, levels of IL-6, TNF- $\alpha$ GM-CSF and MCP-1 were then modulated and maintained at basal level as observed by day 1 post infection (Figures 4 and 5). This could suggest that priming the host with CRX-527 could attenuate cytokine/chemokine production during infection, which has been shown to augment bacterial clearance and improve survival [42].Thirdly, neutrophils that were primed during pre-treatment couldalso be rapidly activated, resulting in immediate production of reactive oxygen species and enhanced phagocytosis upon infection [18]. Such containment might also prevent the cytokine/chemokine storm that was observed in their untreated counterparts.

In conclusion, the model used in this study mimics human pneumonic melioidosis. The course of infection is not restricted to the lung mucosa and can eventually lead to septicaemia if infection is not controlled early and efficiently. CRX-527 non-specifically primes the host's innate immune system. This priming aids in resistance and early containment of the bacteria, while susceptibil- 
ity, as seen in untreated mice, correlates with hyperproduction of cytokines/chemokines from overwhelming bacterial colonization, which progresses to septic shock and eventually death. More importantly, data from this study highlights the potential of CRX-527 and related TLR4-active AGPs as standalone prophylactic or even adjunctive treatment against $\mathrm{Bp}$ infection. Hence, future work will aim to maximize the use of an appropriate AGP as a combination therapy with antibiotics or vaccine adjuvant against melioidosis.

\section{ACKNOWLEDGEMENTS}

This study was supported by a grant from Defence Research \& Technology Office, Ministry of Defence, Singapore. We thank Dr. Jay Evans and Dr. David Johnson from GlaxoSmithKline Vaccines (Hamilton, Montana, USA) for their valuable suggestions in the design of the experiments and critical review of this manuscript.

\section{REFERENCES}

[1] Lazar Adler, N.R., Govan, B., Cullinane, M., et al. (2009) The molecular and cellular basis of pathogenesis in melioidosis: How does Burkholderia pseudomallei cause disease? FEMS Microbiology Reviews, 33, 1079-1099. doi:10.1111/j.1574-6976.2009.00189.x

[2] Inglis, T.J., Rolim, D.B. and Sousa Ade, Q. (2006) Melioidosis in the Americas. The American Journal of Tropical Medicine and Hygiene, 75, 947-954. Cuadros, J., Gil, H., Miguel, J. D., et al. (2011) Case report: Melioidosis imported from West Africa to Europe. The American Journal of Tropical Medicine and Hygiene, 85, 282284. doi:10.4269/aitmh.2011.11-0207

[3] Gan, Y.-H. (2005) Interaction between Burkholderia pseudomallei and the host immune response: Sleeping with the enemy? Journal of Infectious Diseases, 192, 18451850. doi: $10.1086 / 497382$

[4] Gan, Y.-H. (2005) Interaction between Burkholderia pseudomallei and the host immune response: Sleeping with the enemy? Journal of Infectious Diseases, 192, 1845-1850. doi:10.1086/497382

[5] Limmathurotsakul, D. and Peacock, S.J. (2011) Melioidosis: A clinical overview. British Medical Bulletin, 99, 125-139. doi:10.1093/bmb/ldr007

[6] White, N.J. (2003) Melioidosis. Lancet, 361, 1715-1722. doi:10.1016/S0140-6736(03)13374-0

[7] Patel, N., Conejero, L., De Reynal, M., Easton, A., Bancroft, G.J. and Titball, R.W. (2011) Development of vaccines against Burkholderia pseudomallei. Frontiers in Microbiology, 2, 198. doi:10.3389/fmicb.2011.00198

[8] Cheng, A.C. and Currie, B.J. (2005) Melioidosis: Epidemiology, pathophysiology, and management. Clinical Microbiology Reviews, 18, 383-416. doi:10.1128/CMR.18.2.383-416.2005

[9] Wiersinga, W.J., van der Poll, T., White, N.J., Day, N.P. and Peacock, S.J. (2006) Melioidosis: Insights into the pathogenicity of Burkholderia pseudomallei. Nature Re- views Microbiology, 4, 272-282. doi: $10.1038 /$ nrmicro 1385

[10] Propst, K.L., Troyer, R.M., Kellihan, L.M., Schweizer, H.P. and Dow, S.W. (2010) Immunotherapy markedly increases the effectiveness of antimicrobial therapy for treatment of Burkholderia pseudomallei infection. Antimicrobial Agents and Chemotherapy, 54, 1785-1792. doi:10.1128/AAC.01513-09

[11] Powell, K., Ulett, G., Hirst, R. and Norton, R. (2003) G-CSF immunotherapy for treatment of acute disseminated murinemelioidosis. FEMS Microbiology Letters, 224, 315-318. doi:10.1016/S0378-1097(03)00473-7

[12] Cheng, A.C., Limmathurotsakul, D., Chierakul, W., Getchalarat, N., Wuthiekanun, V., Stephens, D.P., Day, N.P., White, N.J., Chaowagul, W., Currie, B.J. and Peacock, S.J. (2007) A randomized controlled trial of granulocyte colony-stimulating factor for the treatment of severe sepsis due to melioidosis in Thailand. Clinical Infectious Diseases, 45, 308-314. doi:10.1086/519261

[13] Kawai, T. and Akira, S. (2010) The role of pattern-recognition receptors in innate immunity: Update on tolllike receptors. Nature Immunology, 11, 373-384. doi:10.1038/ni.1863

[14] Novem, V., Shui, G., Wang, D., et al. (2009) Structural and biological diversity of lipopolysaccharides from Burkholderia pseudomallei and Burkholderia thailandensis. Clinical and Vaccine Immunology, 16, 1420-1428. doi:10.1128/CVI.00472-08

[15] Rowe, D.C., McGettrick, A.F., Latz, E., et al. (2006) Themyristoylation of TRIF-related adaptor molecule is essential for Toll-like receptor 4 signal transduction. Proceedings of the National Academy of Sciences, 103, 62996304. doi:10.1073/pnas.0510041103

[16] Gibbons, H.S., Lin, S., Cotter, R.J. and Raetz, C.R. (2000) Oxygen requirement for the biosynthesis of the S-2-hydroxymyristate moiety in Salmonella typhimurium lipid A. Function of $\mathrm{LpxO}$, a new $\mathrm{Fe}^{2+} /$ alpha-ketoglutarate-depen dent dioxygenase homologue. The Journal of Biological Chemistry, 275, 32940-32949. doi:10.1074/jbc.M005779200

[17] Johnson, A.G. (1994) Molecular adjuvants and immunomodulators: New approaches to immunization. Clinical Microbiology Reviews, 7, 277-289.

[18] Airhart, C.L., Rohde, H.N., Bohach, G.A., et al. (2008) Induction of innate immunity by lipid A mimetics increases survival from pneumonic plague. Microbiology, 154, 2131-2138. doi:10.1099/mic.0.2008/017566-0

[19] Baldridge, J.R., Cluff, C.W., Evans, J.T., et al. (2002) Immunostimulatory activity of aminoalkylglucosaminide 4-phosphates (AGPs): Induction of protective innate immune responses by RC-524 and RC-529. Journal of Endotoxin Research, 8, 453-458.

[20] Lembo, A., Pelletier, M., Iyer, R., et al. (2008) Administration of a synthetic TLR4 agonist protects mice from pneumonic tularemia. The Journal of Immunology, 180, 7574-7581.

[21] Bowen, W.S., Minns, L.A., Johnson, D.A., et al. (2012) Selective TRIF-dependent signaling by a synthetic tolllike receptor 4 agonist. Science Signaling, 5, ra13. 


\section{doi:10.1126/scisignal.2001963}

[22] Cluff, C.W., Baldridge, J.R., Stover, A.G., et al. (2005) Synthetic toll-like receptor 4 agonists stimulate innate resistance to infectious challenge. Infection and Immunity, 73, 3044-3052. doi:10.1128/IAI.73.5.3044-3052.2005

[23] Stover, A.G., Da Silva Correia, J., Evans, J.T., et al. (20 04) Structure-activity relationship of synthetic toll-like receptor 4 agonists. The Journal of Biological Chemistry, 279, 4440-4449. doi:10.1074/jbc.M310760200

[24] Currie, B.J. (2003) Melioidosis: An important cause of pneumonia in residents of and travellers returned from endemic regions. European Respiratory Journal, 22, 542550. doi:10.1183/09031936.03.00006203

[25] Sim, S.H., Liu, Y., Wang, D., et al. (2009) Innate immune responses of pulmonary epithelial cells to Burkholderia pseudomallei infection. PLoS One, 4, Article ID: e7308. doi:10.1371/journal.pone.0007308

[26] Johnson, D.A. (2008) Synthetic TLR4-active glycolipids as vaccine adjuvants and stand-alone immunotherapeutics. Current Topics in Medicinal Chemistry, 8, 64-79. doi:10.2174/156802608783378882

[27] Guthrie, L.A., McPhail, L.C., Henson, P.M. and Johnston Jr., R.B., (1984) Priming of neutrophils for enhanced release of oxygen metabolites by bacterial lipopolysaccharide. Evidence for increased activity of the superoxide-producing enzyme. The Journal of Experimental Medicine, 160, 1656-1671. doi:10.1084/jem.160.6.1656

[28] Sheppard, F.R., Kelher, M.R., Moore, E.E., et al. (2005) Structural organization of the neutrophil NADPH oxidase: Phosphorylation and translocation during priming and activation. Journal of Leukocyte Biology, 78, 1025-1042. doi:10.1189/j1b.0804442

[29] Wiersinga, W.J., Wieland, C.W., Roelofs, J.J. and van der Poll, T. (2008) MyD88 dependent signaling contributes to protective host defense against Burkholderia pseudomallei. PLoS One, 3, Article ID: e3494. doi:10.1371/journal.pone.0003494

[30] Vincent, J.L. (2008) Clinical sepsis and septic shock-definition, diagnosis and management principles. Langenbeck's Archives of Surgery, 393, 817-824. doi:10.1007/s00423-008-0343-1

[31] Blackwell, T.S. and Christman, J.W. (1996) Sepsis and cytokines: Current status. British Journal of Anaesthesia, 77, 110-117. doi:10.1093/bja/77.1.110

[32] Ramnath, R.D., Ng, S.W., Guglielmotti, A. and Bhatia, M. (2008) Role of MCP-1 in endotoxemia and sepsis. International Immunopharmacology, 8, 810-818. doi:10.1016/j.intimp.2008.01.033
[33] Lauw, F.N., Simpson, A.J., Prins, J.M., et al. (1999) Elevated plasma concentrations of interferon (IFN)-gamma and the IFN-gamma-inducing cytokines interleukin (IL)18, IL-12, and IL-15 in severe melioidosis. Journal of Infectious Diseases, 180, 1878-1885. doi:10.1086/315155

[34] Wiersinga, W.J., Wieland, C.W., van derWindt, G.J., et al. (2007) Endogenous interleukin-18 improves the early antimicrobial host response in severe melioidosis. Infection and Immunity, 75, 3739-3746. doi:10.1128/IAI.00080-07

[35] Brown, A.E., Dance, D.A., Suputtamongkol, Y., et al. (1991) Immune cell activation in melioidosis: Increased serum levels of interferon-gamma and soluble interleukin2 receptors without change in soluble CD8 protein. Journal of Infectious Diseases, 163, 1145-1148. doi:10.1093/infdis/163.5.1145

[36] Ulett, G.C., Ketheesan, N. and Hirst, R.G. (2000) Cytokine gene expression in innately susceptible BALB/c mice and relatively resistant $\mathrm{C} 57 \mathrm{BL} / 6$ mice during infection with virulent Burkholderia pseudomallei. Infection and Immunity, 68, 2034-2042. doi:10.1128/IAI.68.4.2034-2042.2000

[37] Barnes, J.L., Ulett, G.C., Ketheesan, N., et al. (2001) Induction of multiple chemokine and colony-stimulating factor genes in experimental Burkholderia pseudomallei infection. Immunology and Cell Biology, 79, 490-501. doi:10.1046/j.1440-1711.2001.01038.x

[38] Liu, B., Koo, G.C., Yap, E.H., Chua, K.L. and Gan, Y.H. (2002) Model of differential susceptibility to mucosal Burkholderia pseudomallei infection. Infection and Immunity, 70, 504-511. doi:10.1128/IAI.70.2.504-511.2002

[39] Tan, G.Y., Liu, Y., Sivalingam, S.P., et al. (2008) Burkholderia pseudomallei aerosol infection results in differential inflammatory responses in BALB/c and C57B1/6 mice. Journal of Medical Microbiology, 57, 508-515. doi:10.1099/jmm.0.47596-0

[40] Lertmemongkolchai, G., Cai, G., Hunter, C.A. and Bancroft, G.J. (2001) Bystander activation of CD8+ T cells contributes to the rapid production of IFN-gamma in response to bacterial pathogens. The Journal of Immunology, 166, 1097-1105.

[41] Santanirand, P., Harley, V.S., Dance, D.A., Drasar, B.S. and Bancroft, G.J. (1999) Obligatory role of gamma interferon for host survival in a murine model of infection with Burkholderia pseudomallei. Infection and Immunity, 67, 3593-3600.

[42] Wheeler, D.S., Lahni, P.M., Denenberg, A.G., et al. (2008) Induction of endotoxin tolerance enhances bacterial clearance and survival in murinepolymicrobial sepsis. Shock, 30, 267-273. 